Philosophie ANTIQUE

\section{Philosophie antique}

Problèmes, Renaissances, Usages

$18 \mid 2018$

L'athéisme antique

\title{
Andrea FALCON, Aristotelismo
}

\section{Marie-Noëlle Ribas}

\section{OpenEdition \\ Journals}

Édition électronique

URL : https://journals.openedition.org/philosant/1099

DOI : 10.4000/philosant.1099

ISSN : 2648-2789

Éditeur

Éditions Vrin

\section{Édition imprimée}

Date de publication : 1 novembre 2018

Pagination : 288-290

ISBN : 978-2-7574-2372-1

ISSN : $1634-4561$

\section{Référence électronique}

Marie-Noëlle Ribas, «Andrea falcon, Aristotelismo », Philosophie antique [En ligne], 18| 2018, mis en ligne le 01 novembre 2018, consulté le 03 décembre 2022. URL : http://journals.openedition.org/ philosant/1099; DOI : https://doi.org/10.4000/philosant.1099

\section{(c) $)(9)$}

Creative Commons - Attribution - Pas d'Utilisation Commerciale - Pas de Modification 4.0 International - CC BY-NC-ND 4.0

https://creativecommons.org/licenses/by-nc-nd/4.0/ 
et celui de la responsabilité morale du lemme aĩt- dissolvant le rôle singulier qu'a joué historiquement l'Éloge d'Hélène dans la constitution philosophique du problème de la responsabilité. Le respect à tout prix du principe d'invariance dans la traduction de $\lambda$ ó $\gamma$ os pose un problème similaire, d'autant que le terme, tel qu'il apparaît chez Gorgias, résiste à toute polarisation : il est nécessairement « discours » au paragraphe 8 , puisqu'il

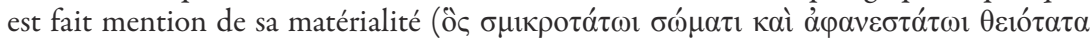
है $\gamma \gamma \alpha \dot{\alpha} \pi 0 \tau \varepsilon \lambda \varepsilon \hat{\imath})$, impérativement « argument » au paragraphe 15, alors qu'il introduit

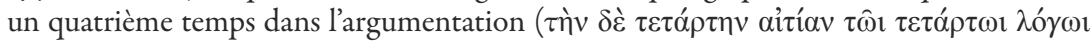

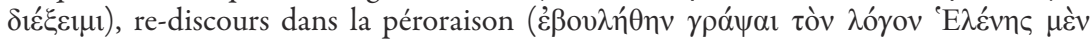

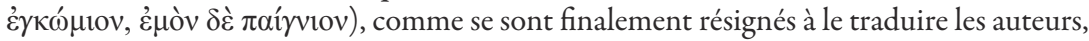
de guerre lasse. En dépit de la qualité de la traduction, l'ensemble des commentaires ci-dessus ainsi que la restriction des notes à leur seule fonction référentielle relative au dialogue de Platon laisse au lecteur désireux de découvrir un texte tout à fait singulier dans l'histoire de la philosophie et de la rhétorique l'amère impression de n'avoir accès ici quà une annexe du Gorgias de Platon. Notons également la traduction de íßpí $\sigma \theta$, í $\beta \rho_{i ́ \sigma} \alpha \varsigma, \dot{v} \beta \rho ı \sigma \theta \varepsilon i \sigma \alpha$ par « viol » qui apporte une précision bienvenue au texte - car c'est probablement ce que nous devons, aujourd'hui, lire sous ce passage ; mais peut-être aurait-il fallu apporter cette précision dans les notes, le « viol » étant un concept tout à fait étranger à la pensée et à la réalité grecques ( $c f$. S. Deacy et K. F. Pierce (éd.), Rape in Antiquity, Sexual Violence in the Greek and Roman Worlds, London, 1997).

Ces quelques réserves mises à part, cette double traduction reste un ouvrage précieux susceptible d'inciter à lire ou à relire ces deux chefs-d'œuvre de la philosophie et de la rhétorique que sont l'Éloge d'Hélène et le Gorgias de Platon et nous remercions les auteurs pour cela.

Julie Tramonte

Andrea Falcon, Aristotelismo, Turin, Piccola Biblioteca Einaudi, 2017, 150 p., ISBN 978-88-06-23112-5.

Dans cet ouvrage, A. Falcon propose une histoire de l'aristotélisme qui examine la présence et la fortune de la pensée d'Aristote dans le monde antique. L'originalité du livre ne réside pas dans son objet, comme le souligne l'auteur en mentionnant dès l'introduction le travail colossal publié en 1973 par Paul Moraux sur l'aristotélisme, d'Andronicos de Rhodes à Alexandre d'Aphrodise (Der Aristotelismus bei den Griechen, von Andronikos bis Alexander von Aphrodisias, Berlin, 1973). Elle réside dans l'ampleur d'une étude qui ne réduit pas l'histoire de l'aristotélisme à celle de l'école aristotélicienne et qui s'étend de la mort d'Aristote vers 322 av. J.-C. jusqu'à l'Antiquité tardive.

Le premier chapitre considère la période allant de la mort d'Aristote au I ${ }^{\text {er }}$ s. apr. J.- C., dont la caractéristique principale est une relative absence de la référence explicite à Aristote, exception faite de quelques renvois à des œuvres perdues, comme le Protreptique ou le dialogue Sur la Justice. Si certaines études expliquent ce silence relatif en distinguant l'Aristote exotérique, connu à l'époque hellénistique, du nôtre, qui est celui des textes ésotériques, A. Falcon met en lumière une situation plus complexe. Non seulement quelques œuvres de l'école circulent déjà à cette époque, comme en témoigne un fragment de lettre qui mentionne les Analytiques et les écrits naturels (PHerc. 1005 = fr. 127 Arrighetti), mais il faut apprécier la présence d'Aristote à partir des références implicites qui y sont faites. Reprenant le principe exégétique de Francis H. Sandbach qui apprécie l'impact d'Aristote sur la philosophie hellénistique en montrant que les thèses 
hellénistiques s'expliquent plus facilement à partir de lui, A. Falcon expose par exemple comment la doctrine épicurienne des minima répond indirectement à l'argumentation du sixième livre de la Physique d'Aristote. La méthode, féconde dans le cas d'Épicure, montre toutefois ses limites, lorsque F. Sandbach se croit autorisé à douter à partir d'elle de l'influence d'Aristote sur les premiers stö̈ciens. Les similitudes qu'il constate dans les domaines physique et éthique entre eux et Aristote ne seraient quapparentes et s'expliqueraient par une origine académique commune. Les deux principes stö̈ciens $\mathrm{du}$ monde physique que sont la Raison ou Zeus comme principe actif, et la matière comme principe passif, rappellent l'hylémorphisme d'Aristote parce qu'ils auraient tous deux leur source dans le Timée de Platon. À la suite de l'article « Aristotle and the Stoa » que Thomas Benatouil a consacré à la question en 2016 (A. Falcon éd., Brill's Companion to the Reception of Aristotle in Antiquity, Leiden, p. 56-75, DOI : 10.1163/9789004315402_005), A. Falcon souligne que la thèse de Sandbach ignore le rapport dialectique qui existe entre Aristote et les stoïciens, comme le montre l'examen qu'il consacre à la conception chrysippéenne du mélange (p. 11). La philosophie hellénistique retrouve des thèmes de la tradition présocratique qu'elle révise à la lumière des critiques formulées par Aristote, présenté comme un membre de l'Académie, et sur ce point la philosophie stoïcienne ne fait pas exception.

Les deuxième et troisième chapitres consacrés à l'époque post-hellénistique interrogent le sens à donner au « retour à Aristote » qui caractérise la période, sachant que la redécouverte des livres d'Aristote ou que le travail d'organisation des œuvres opéré par Andronicos de Rhodes ne suffisent pas à l'expliquer. Après avoir retracé les étapes supposées conduire à l'oubli des textes d'Aristote, puis à leur redécouverte au $\mathrm{I}^{\text {er }}$ s. av. J.-C., A. Falcon montre que la réorganisation du corpus aristotélicien effectuée par Andronicos n'est sans doute pas étrangère au succès des Catégories. L'œuvre d'Aristote doit commencer par la logique, et la logique par le traité des Catégories, qui est commenté, paraphrasé, critiqué, dans les traditions péripatéticienne comme stoïcienne. En étudiant comment Boéthos de Sidon utilise le texte des Catégories pour repenser l'hylémorphisme d'Aristote, qui est une doctrine extérieure à ce traité, l'auteur prend soin de ne pas réduire le retour post-hellénistique d'Aristote à un retour aux Catégories. Même si la conception de Boéthos est peu conforme à celle d'Aristote, celui-ci « pense la doctrine hylémorphique avec (et non contre) Aristote » (p. 34). La négation de la réalité des universaux, la réduction de la forme à un accident de la matière ou la thèse que la matière est le sujet ultime de la prédication sont trois éléments d'une ontologie qu'une lecture libre des Catégories rend possible. Boéthos de Sidon qui travaille à gommer les divergences entre aristotélisme et stoïcisme est exemplaire de ces auteurs qui, dans un contexte d'attention renouvelée au texte, cherchent dans les œuvres d'Aristote les ressources conceptuelles pour développer des alternatives crédibles à la philosophie stoïcienne alors dominante. Par une série d'études précises portant aussi bien sur l'éthique péripatéticienne au $\mathrm{I}^{\mathrm{er}}$ s. av. J.-C. que sur l'école péripatéticienne, A. Falcon invite son lecteur à abandonner une lecture finaliste de l'aristotélisme qui présente son histoire comme un progrès vers une plus grande authenticité dans la lecture d'Aristote. P. Moraux parlait en ce sens de la « volonté d'orthodoxie » qui anime les principaux acteurs de l'aristotélisme post-hellénistique et présentait ceux qui ne visent pas cet objectif comme des penseurs hétérodoxes. En abandonnant l'idée d'une lecture authentique d'Aristote, A. Falcon prétend ressaisir l'aristotélisme post-hellénistique dans sa complexité, c'est-àdire dans sa pluralité. Xénarque de Séleucie qui critique la doctrine de la quintessence n'est pas un adversaire à l'intérieur de la pensée d'Aristote comme le soutient P. Moraux. 
Être en désaccord partiel avec Aristote n'interdit pas aux philosophes de l'époque d'en revenir à lui pour trouver dans ses écrits les éléments d'une philosophie aristotélicienne. La lecture d'Aristote proposée par Xénarque n'est pas plus créative ou moins fidèle que celles avancées par Boéthos ou Alexandre d'Aphrodise. Loin d'être dépourvue d'originalité, la philosophie post-hellénistique est ce moment où une lecture libre des textes d'Aristote permet à certains de rivaliser avec les philosophes stoïciens, qu'il s'agisse pour les péripatéticiens de développer une philosophie aristotélicienne ou pour certains platoniciens d'inscrire Aristote et Platon dans une même tradition philosophique. En intégrant la pensée aristotélicienne au sein d'une tradition académique qui remonterait à Pythagore, Antiochus d'Ascalon ou Eudore d'Alexandrie entendent promouvoir une forme dogmatique de platonisme. Chez eux, comme chez Jamblique qui établit l'origine pythagoricienne de la doctrine des Catégories, le retour à Aristote n'est jamais qu'un retour aux Anciens. Cet éclairage permet d'interpréter le retour à Aristote autrement que comme la conséquence directe de la redécouverte de ses écrits, puisqu'il serait l'effet d'un phénomène plus large qu'A. Falcon identifie à un retour aux maîtres du passé.

Le quatrième chapitre montre comment la réception d'Aristote dans l'Antiquité tardive se réalise entièrement à l'intérieur de la tradition platonicienne, dans un contexte où la pensée de Platon constitue le point de départ et d'arrivée de toute réflexion. À la différence d'un Alcinoos ou d'un Apulée, des auteurs comme Proclus ou Damascius ne cherchent pas à faire passer pour platoniciens les éléments qu'ils empruntent à Aristote. Ils essaient plutôt de les concilier avec la philosophie de Platon. À la suite de Riccardo Chiaradonna, A. Falcon examine la forme de conciliation que la critique plotinienne des Catégories rend nécessaire. Sans nier la portée ontologique des Catégories comme le font les stoïciens, Plotin, qui établit que le langage dont s'occupe le traité d'Aristote est celui qui s'applique aux réalités sensibles, prépare la lecture de Porphyre. Dans son introduction à l'enseignement des catégories qu'est l'Isagoge, Porphyre soutient qu'elles sont des catégories linguistiques avant d'être des catégories de l'être dans sa dimension sensible, ce qui la rend compatible avec la thèse métaphysique de Platon d'une priorité des substances intelligibles. Avec Porphyre, la confrontation au texte des Catégories devient le passage obligé de toute initiation à la philosophie de Platon, comme ce sera le cas par exemple dans l'école d'Athènes où la logique constitue le point de départ d'un parcours qui culmine avec l'étude du principe divin. Qu’Aristote soit un disciple de Platon et que sa pensée fasse partie intégrante du platonisme est une idée que les philosophes de l'Antiquité tardive partagent malgré leurs différences. Le dernier chapitre du volume, qui restitue les vicissitudes liées à la transmission de la biologie, de la logique et de la noétique aristotéliciennes, finit de nous convaincre que l'adaptation de certains aspects de la pensée d'Aristote au contexte platonicien se fait au détriment d'autres pans de sa doctrine qui nous paraissent essentiels aujourd'hui, comme ses recherches sur le vivant.

On ne peut que recommander la lecture de cette histoire de l'aristotélisme qui nous découvre un Aristote qui ne nous est pas familier. Perçu par certains contemporains comme un rival, critiqué par ses successeurs immédiats pour sa personne comme pour son enseignement, Aristote est loin d'être « le Maître de ceux qui savent » et le philosophe par excellence que décrit Dante au quatrième chant de la Divine Comédie. L'ouvrage d'A. Falcon a le mérite de montrer que sa présence et celle de sa pensée n'en sont pas moins décisives dès l'époque hellénistique, où les problèmes aristotéliciens structurent nombre de débats. 